\title{
Human-Robot Collaboration: Twofold Strategy Algorithm to Avoid Collisions Using ToF Sensor
}

\author{
Rafiq Ahmad and Peter Plapper
}

\begin{abstract}
The importance of Human Robot Interaction to complement human skills in a manufacturing environment with industrial robots increases the concerns over safety of human and the robot. It is necessary to identify collision risks and avoid them otherwise production stops may cost a huge amount to the industry. A robot working at manufacturing facility should be able to predict potential collisions and must be able to prevent i.e. react automatically for safe detour around obstacle/human. Currently, industrial robots are able to detect collisions after a real contact but the existing proposals for avoiding collisions are either computationally expensive or not very well adapted to human safety. The objective of this paper is to provide intelligence to the industrial robot to predict collision risks and react automatically without stopping the production in a static environment. The proposed approach using Time of Flight (TOF) camera, provides decision regarding trajectory correction and improvement by shifting robot to a secure position. The application presented in this paper is for safe KUKA robot trajectory generation in peg-in-hole assembly process in the laboratory context.
\end{abstract}

Index Terms-Robot path planning, safe robot trajectory generation, collision detection and avoidance, vision based system, time of flight (ToF) sensor.

\section{INTRODUCTION}

The vision of future semi-automated robotized assembly requires industrial robots to cooperate with human workers in order to perform tasks according to their individual strength. Conventional industrial assembly is based on the fact that robots are separated from humans by fences, light guards or gates and if not then they may continuously touch a human working nearby with limited but unbearable force in industrial environment. During manufacturing and assembling, human and machine elements may act as obstacles in the planned robot trajectory. A collision of robot with a human, maybe fatal for human whereas, a collision with a machine element may also cost a huge amount to the company for a manufacturing breakdown or damage to the robot.

In order to avoid human injuries, legal and standardization activities permit the cooperation of human and robots under specific constraints such as limiting the force. First robot manufacturer implemented these legal requirements in

Manuscript received March 19, 2015; revised June 1, 2015. This work was supported by IEE industry and sponsored by FNR-AFR Luxembourg funding.

R. Ahmad is with the University of Luxembourg, L-1359 Luxembourg, on leave from the CECOS University of IT and Emerging Sciences, Pakistan (e-mail: rafiq.ahmad@uni.lu).

P. Plapper is with the University of Luxembourg, Luxembourg, L-1359 Luxembourg (e-mail: peter.plapper@uni.lu). commercially available robots [1], which are a prerequisite for Human-Robot Collaboration. Most robots are programmed to stop when a collision occurs based on tactile force sensors. This unintended and annoying contact will interrupt the work flow of the robot as well as the human and thus must be avoided, otherwise it may cause production stops and extended costs for the company. Some researchers adjust the motion of the robot as a reaction on intruding obstacles (e.g. human worker) [2], [3]. A well-known method to react to intruding obstacles is the artificial potential field [2]. The drawback of the method is that robot may get stuck in a local minimum. A randomized-roadmap [4] based path planning in the Cartesian coordinate system is also used, which provides information of the Tool Center point collisions only. Different solutions were investigated [5] to adapt robot to the changing environment but they are not suitable for real-time unknown and changing obstacles. The reason behind this is that a standard safe sensor technology is still missing, and most of the existing methods needs robustness in terms of computation time to adapt itself to safe continuous path planning.

With optical camera systems [6], stationary surveillance zones can be defined. An intrusion into these zones will slow down the robot motion or stops the robot completely. 2D vision cameras are good for objects detection and identification but are sensitive to light and industrial environment. These cameras are also not suitable computationally for path planning in a 3D environment. In order to enhance the vision capabilities of the robot to interact effectively with human, Time-of-Flight (ToF) depth sensor is used because of its many advantages [7], [8] such as they are not affected by the intensity differences between images and provide immediate depth information of the environment. ToF sensors seems to be an effective solution, which rapidly perceive the 3D environment and are less affected by light conditions. For instance, two Kinect camera sensors, which operate with infrared illumination of the scene and use triangulation to observe distances to the obstacles with reduced occlusion [9]. The speed of the robot is adjusted depending on the distance of the approaching obstacle with a linear slope. However, a correction of the path to detour around the obstacle is not provided yet. A solution is therefore necessary, which takes into account the 3D information from the scene and provide rapid decision for robot path planning.

The approach presented in the next section will provide a twofold approach to avoid collision, i.e. 1) avoid collision with human by proper scheduling and 2) detour robot around the obstacle to avoid collision automatically. 


\section{TwOFOld STRATEGY TO Avoid COLLISIONS}

A twofold strategy is presented for robot safe working environment, depending on the scenario on hand i.e. workplace sharing or workplace and time sharing systems [10]. A single ToF sensor is installed to monitor a peg-in-hole process environment where a KUKA robot is working and a human worker or any static object may act as obstacle. Collision is either prevented by proper scheduling or detected and avoided by giving proper intelligence to the robot in order to decide its safe trajectory. A Robot Path Planning ( RPP=R2P) algorithm based on two fold strategy is the contribution of the paper, which intelligently decides safe detour around obstacles, i.e. static object as initial application in this paper. In the case of collision, the algorithm presented finds a safe solution either in the form of proper scheduling or an effective intelligent decision to detour the obstacle with a proper distance.

\section{A. Proper Scheduling}

A proper schedule is required to avoid collisions between human and a robot in a shared workplace, workplace and time sharing scenario. Data from the ToF sensor mounted on top of the scene helps to monitor simultaneous work and detecting the potential risk of collision. If a human is working, the robot is programmed to shift to another location and if a robot is working, a human has to work at another station.

\section{B. Proper Intelligence}

In the case of robot and human working together in a common workplace and time sharing environment, human safety is the top priority. Robot working with human should be intelligent enough to not only detect collisions with human or other obstacles but also be able to plan a safe trajectory. This intelligence in decision process is important to avoid human injury, equipment damage, reduce idle time of the system by avoiding collisions with the obstacle/human.

\section{Robot Path Planning (R2P) Algorithm}

As said, to insert images in Word, position the cursor at the insertion point and either use Insert |Picture| From File or copy the image to the Windows clipboard and then Edit Paste Special | Picture (with "Float over text" unchecked).

A Robot Path Planning (R2P) Algorithm is presented to plan robot safe trajectory in a static environment as an initial application. Although, R2P algorithm is planned to generate safe trajectories during Human-Robot Interaction by detecting and avoiding collision with human, but as per initial application, results in this paper is based on static obstacle (machine part, imaginary obstacle etc.). A robot carrying a peg-in-hole process is taken as case study as shown in Fig. 1. The tip point of peg is considered for trajectory generation, whereas trajectory in focus is a transversal trajectory between three functional work points.

An assembly scene is considered where a robot has to follow a pre-programmed trajectory. A 3D-MLI sensor [10] installed on the table captures the depth information from the scene and localizes different object in the 3D working environment. While knowing different items like work-points, initial planned trajectory, the scene information in the presence and absence of obstacle, the $\mathrm{R} 2 \mathrm{P}$ algorithm detailed in the program code below, calculates the safe robot trajectory. Thus avoiding static and unknown obstacles in the scene by verifying robot manipulator planned trajectory.

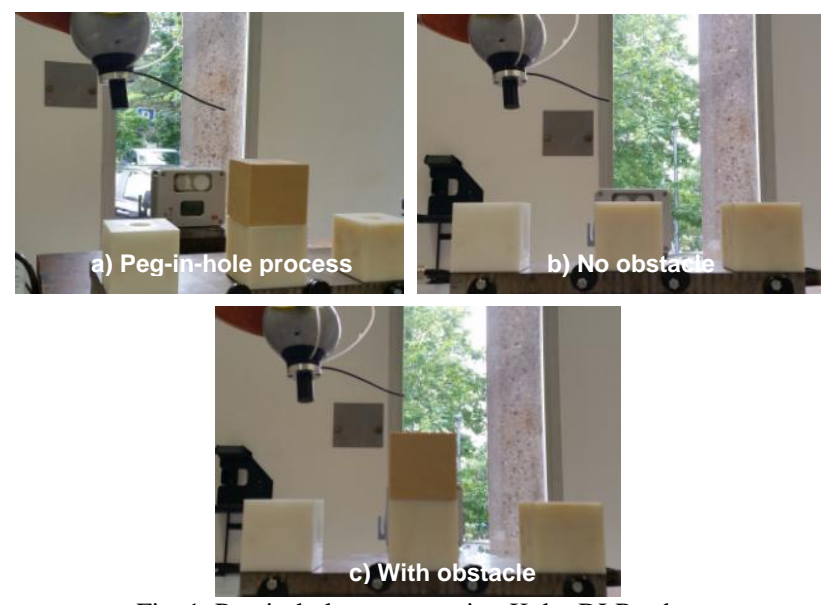

Fig. 1. Peg-in-hole process using Kuka-DLR robot.

In the case of potential risk of collision, a safe trajectory is devised by diverting the robot to a secure position. Initial environment information are collected from the scene in a depth information Matrix A where no obstacle is present as shown in Fig. 2. This image matrix is stored as a reference information matrix where the robot trajectory is already programmed. The actual scene information are updated to the algorithm and the new depth information in the presence of a static obstacle is stored as a new image Matrix B. The two matrices are then subtracted and a new Matrix $\mathrm{C}$ is obtained, which provides information about new objects in the scene.
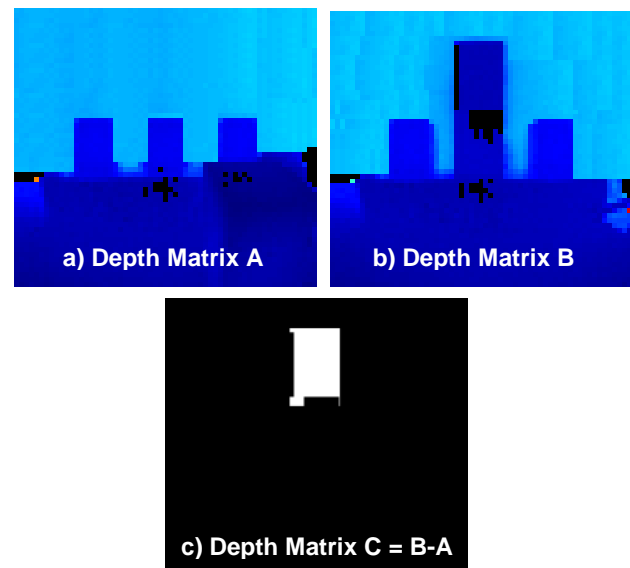

Fig. 2. Depth information matrices from the scene (from 3D-MLI sensor).

The already programmed trajectory is then verified on Matrix $\mathrm{C}$ by checking each trajectory next point. In the presence of obstacle, human/object, the approach presented diverts robot to another working point by straight line movement. Once another collision is detected in the new decided path, the robot is shifted to a new secure location in order to detour the obstacle.

Secure points are generated by detecting the height of obstacle in the scene and defining a row of points above the obstacle in the space. Once secure points are generated then robot is shifted to a secure position by selecting one secure position using minimum distance criteria from the current point to secure point and from the secure point to the final goal point. Once a secure point is selected the trajectory is 
verified again as per the procedure explained above. Every time a collision is detected the secure point's matrix remains the same as this paper assumes fixed obstacle and constant robot speed. Each secure point selected is stored in the database and finally the safe trajectory is generated. The safe trajectory obtained is then improved by removing the unnecessary trajectory points and finally the improved safe trajectory is send back to the Robot using Fast-Research-Interface (FRI) for safe assembling scenario application.

R2P Program (C++): Human-Robot-Collaboration / Collision detection and avoidance

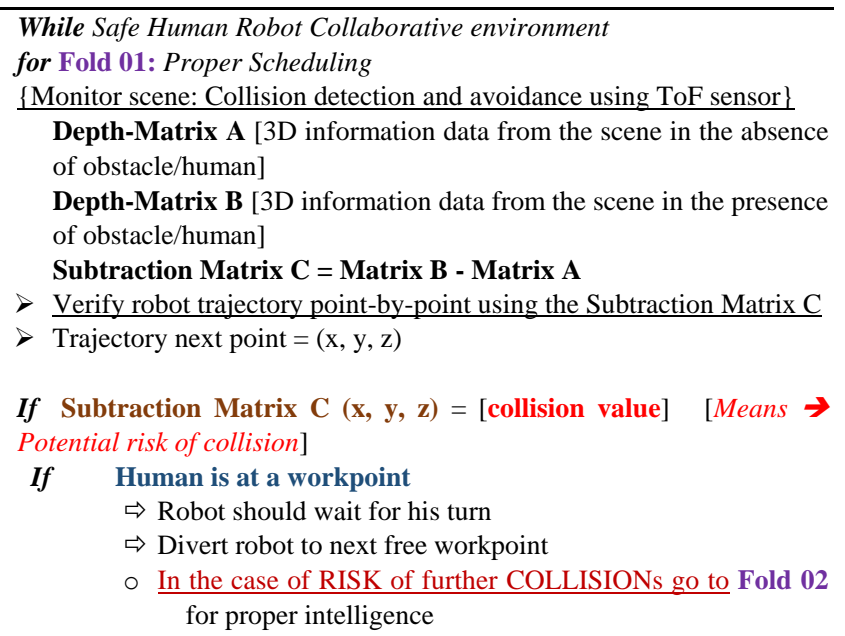

elseif Robot is at workpoint

$\Rightarrow$ Human should wait until robot finish his working

$\Rightarrow$ Divert human to next free workpoint

- In the case of RISK of further COLLISIONs go to Fold 02 for proper intelligence

else Static obstacle present at workpoint

Divert Robot to next free workpoint, go to Fold 02 for proper intelligence end

end

for Fold 02: Proper Intelligence

\{Verify robot trajectory point-by-point using the Subtraction Matrix C

$>$ Trajectory next point $=(\mathrm{x}, \mathrm{y}, \mathrm{z})$

If Subtraction Matrix $\mathbf{C}(\mathbf{x}, \mathbf{y}, \mathbf{z})=$ [collision value $][$ Means $\rightarrow$ Potential risk of collision]

$\Rightarrow$ Identify the obstacle/human location in the Subtraction Matrix C

$\Rightarrow$ Find secure points above the obstacle to divert robot motion to another workpoint

- Check if each point obtained has a pixel value equal to the background

- Select the best safe point using minimum distance criteria:

- Distance $=$ distance from current point to secure point + distance from current point to next workpoint i.e. goal target

$\Rightarrow(\mathbf{x}, \mathbf{y}, \mathbf{z})=\left(\mathbf{x}_{\text {safe, }}, \mathbf{y}_{\text {safe, }}, \mathbf{z}_{\text {safe }}\right)$ [Safe point above the obstacle]

$\Rightarrow$ Plan trajectory from this point onward to reach the next workpoint

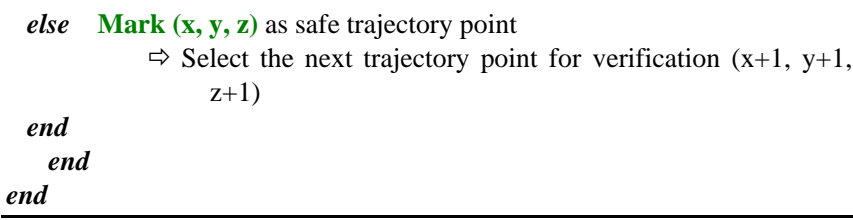

\section{RESULTS AND DISCUSSIONS}

As proper scheduling and collaboration may avoid collisions between a human and worker to a large extent as shown in Fig. 3. An expected collision between a human and robot working in the same area is detected by the ToF camera and robot is rescheduled to another working location. This scenario is generated in V-REP software in order to show an example for proper scheduling but more results in this paper are based on R2P algorithm application on real scenario from the robot peg-in-hole process as shown in Fig. 1 previously, where collision will be detected and avoided with an unknown static obstacle.

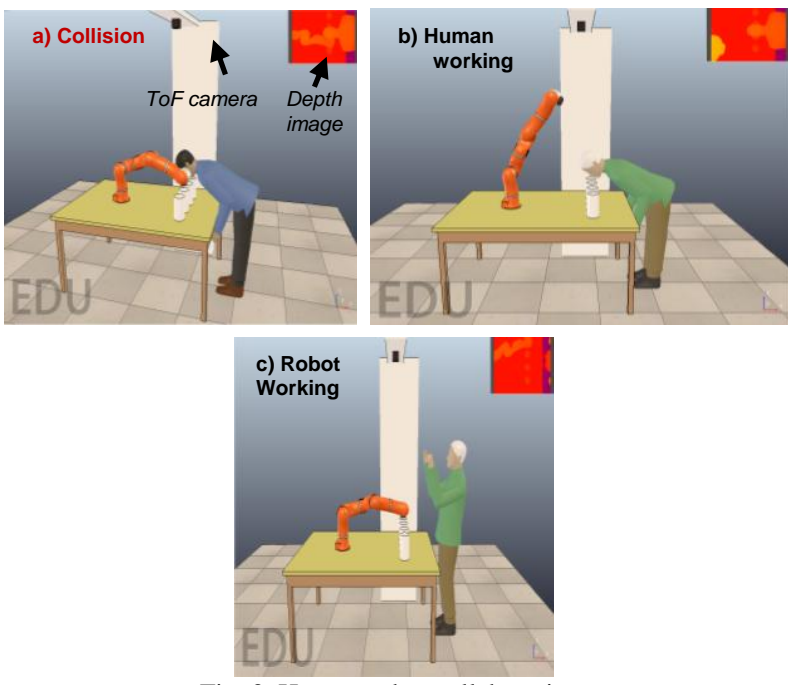

Fig. 3. Human-robot collaboration

A robot programmed initially to peg in the first hole and move onwards to second and third hole. While moving from the first hole to the second, the ToF sensor detects an unknown and un-programmed obstacle (a plastic cube) present at the second hole. The algorithm switches the robot trajectory from first hole directly to third hole by scheduling for a free work point. The R2P algorithm then detects the collision in the new straight line trajectory from first hole to last hole and finds safe trajectory detour to avoid the obstacle. A safe trajectory is generated by detecting safe points above the obstacle to divert the robot as shown in Fig. 4(a). At each point the algorithm finds way for the robot to move straight to the goal point but finds the obstacle again and is retracted to the secure points already defined. The process continues until the final goal point is reached. The trajectory obtained is then improved to reduce the back and forth motion toward/from obstacle, unnecessary points are deleted and new safe improved trajectory is generated as shown in Fig. 4(b).
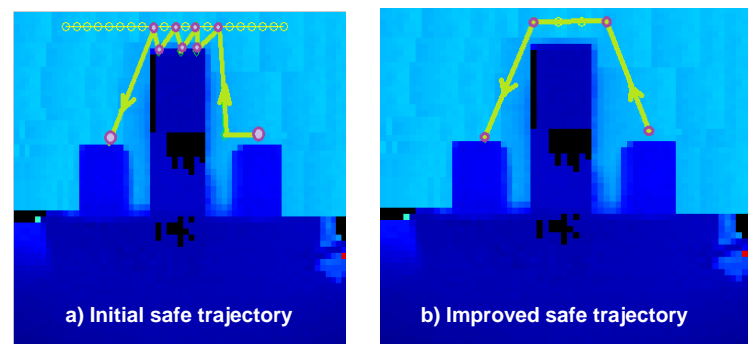

Fig. 4. Safe and automated trajectory solutions (Generated in $\mathrm{C}++$, Computation time: $<1 \mathrm{sec})$. 
In future, the developed approach will be adapted to more complex scenarios, dynamic obstacle detection (human) and occlusion issues will be solved using two sensors.

\section{CONCLUSION}

A two fold strategy is presented to automatically generate safe path for robot trajectory based on data from ToF sensor. A Robot Path Planning (R2P) is developed to detect potential collision risk by ToF sensor data analysis and providing a proper scheduling to decide another working location for the robot. While following the new path to go to the next collision a potential collision (if any) is again detected in the same manner and R2P algorithm generates a safe trajectory detour around the obstacle to reach the final goal. The strategy presented is validated with a peg-in-hole process where collision is avoided with a static obstacle successfully. In future, the approach presented will be applied to real Human Robot Collaborative scenarios.

\section{ACKNOWLEDGMENT}

Authors would like to thank IEE industry for their support in providing 3D-MLI sensor and also for their technical support regarding its installation and processing. Authors would also like to thank FNR for funding the research project Authors would also like to thank Raphael Hinger for his technical help in the demonstration of the results.

\section{REFERENCES}

[1] S. Haddadin, A. Albu-Schäffer, and G. Hirzinger, "Requirements for safe robots: Measurements, analysis and new insights," The International Journal of Robotics Research, vol. 28, pp. 1507-1527, 2009.

[2] O. Khatib, "Real-time obstacle avoidance for manipulators and mobile robots," Robotics and Automation, pp. 500-505, 1985.

[3] A. De Luca and F. Flacco, "Integrated control for pHRI: Collision avoidance, detection, reaction and collaboration," in Proc. 4th IEEE RAS \& EMBS International Conference on Biomedical Robotics and Biomechatronics (BioRob), pp. 288-295, 2012.

[4] T. Gecks and D. Henrich, "Sensor-based online planning of timeoptimized paths in dynamic environments," in Proc. GWR09 German Workshop on Robotics, Braunschweig, Germany, 2009.

[5] B. Siciliano and O. Khatib, Handbook of Robotics, Springer Berlin Heidelberg, 2008.

[6] C. Thomas, F. Busch, B. Kuhlenkoetter, J. Deuse, "Process and human safety in human-robot-interaction - A hybrid assistance system for welding applications," Robotics and Applications, pp. 112-121, 2011.

[7] S. Foix, G. Alenya, and C. Torras, "Lock-in time-of-flight (ToF) cameras: a survey," Sensors Journal, IEEE, vol. 11, no. 9, pp. 1917-1926, 2011.

[8] F. Garcia Becerro, "Sensor fusion combining 3-D and 2-D for depth data enhancement," PhD Thesis, University of Luxembourg, 2012.

[9] D. Henrich and T. Gecks, "Multi-camera collision detection between known and unknown objects," in Proc. ACM/IEEE International Conference on Distributed Smart Cameras, p. 1-10, 2008.

[10] J. Kruger, T. K. Lien, and A. Verl, "Cooperation of human and machines in assembly lines," CIRP Annals - Manufacturing Technology, vol. 58, pp. 628-646, 2009.

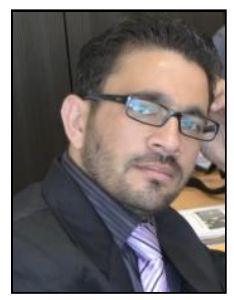

Rafiq Ahmad was born in Swat, Pakistan on $14^{\text {th }}$ March 1983. He got his $\mathrm{PhD}$ in mechanical engineering from IRCCyN, Ecole Cenrale de Nantes, France, in September 2012. His received his master's degree in knowledge integration in mechanical engineering design and production from Ecole Nationale Supérieure d'Arts et Métiers (ENSAM) Paris, France in 2009. His primary four years bachelor's degree was in mechanical engineering from the University of Engineering and Technology Peshawar, Pakistan in 2007. His major field of study and research includes CNC multi-axis machine tool intelligent manufacturing. Knowledge and vision based planning and decision systems, Industrial automation, Industrial and mobile robots planning systems.

Since Sept 2012 he worked as an assistant professor at CECOS University for a year and then obtained a Luxembourgish FNR funding to work as a senior researcher in the University of Luxembourg. He has also participated in various research and development projects at Ecole Centrale de Nantes, France. He got his first insight into the research and academic world after Bachelor's degree where he served as a research assistant at Gulaam Ishaq Khan Institute of Engineering and Technology, Topi, Pakistan.

Dr Rafiq is a member of ASME, International Institute of Engineers and Pakistan Society of Mechanical Engineers. He is also an active reviewer in various conferences and journals that includes: Computer-Aided Design; International Journal of Precision Engineering and Manufacturing; International Journal of Computer Integrated Manufacturing; Chinese Optics Letters Journal; International Journal of Engineering Education; ASME Conference; ICCMA Conference, HSM conference.

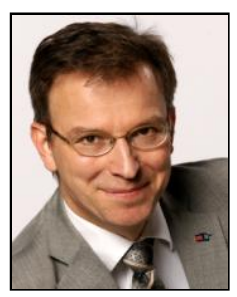

Peter Plapper was born in Mannheim, Germany on September 16th 1963. In 1986 he completed his studies on mechanical engineering / design at TU Kaiserslautern with the degree Dipl.-Ing. His doctoral thesis at the laboratory of tool machines (WZL) of RWTH Aachen, Germany was awarded with the Borchers Medal for scientific excellence in 1993.

Since 1994, he worked for Adam Opel and General Motors in different management positions in Manufacturing Engineering (ME) with increasing responsibility. He developed innovative production technologies, implemented tool machines and coordinated the refurbishment of robotic assembly lines. From 1998 until 2002 he joined the tech center of GM in Michigan, USA where he shaped the global manufacturing strategy for Body Shop and General Assembly. During his industrial career he worked on many different robot applications, led the installation of assembly lines all European GM vehicle plants and was responsible as the head of manufacturing engineering for the equipment of all shops in plant Russelsheim. Following his assignment as a manager advanced technologies europe Peter Plapper was appointed in 2010 full-professor for manufacturing engineering to the University of Luxembourg.

Prof. Plapper is member of AIM (European Academy of Industrial Management), VDI (Verein Deutscher Ingenieure), and Luxembourg Materials and Production Cluster Steering Committee. Since 2014 he is the DIRECTOR of the new Master program "Master of Science in Engineering - Sustainable Product Creation". For the current list of publications。 